\title{
Effect of graphene nanoplatelets on the dielectric permittivity and segmental motions of electrospun poly(ethylene-co-vinyl alcohol) nanofibers
}

JD. Badia',2, R. Teruel-Juanes ${ }^{2}$, Y. Echegoyen ${ }^{3,4}$, S. Torres-Giner ${ }^{3}$, J.M. Lagarón $^{3}$ and A. Ribes-Greus ${ }^{2, *}$

OPEN ACCESS VERSION FROM POST-PRINT

Citation: J.D. Badia, R. Teruel-Juanes, Y. Echegoyen, S. Torres-Giner, J.M. Lagarón, A. Ribes-Greus, Effect of graphene nanoplatelets on the dielectric permittivity and segmental motions of electrospun poly(ethylene-co-vinyl alcohol) nanofibers, Polymer Degradation and Stability, 183 (2021), 109404,

https://doi.org/10.1016/j.polymdegradstab.2020.109404.

Available at:

(https://www.sciencedirect.com/science/article/pii/S0141391020303335) 


\title{
Effect of graphene nanoplatelets on the dielectric permittivity and segmental motions of electrospun poly(ethylene-co-vinyl alcohol) nanofibers
}

\author{
JD. Badia',2, R. Teruel-Juanes ${ }^{2}$, Y. Echegoyen ${ }^{3,4}$, S. Torres-Giner ${ }^{3}$, J.M. \\ Lagarón $^{3}$ and A. Ribes-Greus ${ }^{2, *}$ \\ ${ }^{1}$ Department of Chemical Engineering, School of Engineering, Universitat de València (UV). Av de les \\ Universitats, 46100 Burjassot, València, Spain \\ ${ }^{2}$ Technological Institute of Materials (ITM), Universitat Politècnica de València (UPV). Camino de Vera, s/n, \\ 46022 València, Spain \\ ${ }^{3}$ Novel Materials and Nanotechnology Group, Institute of Agrochemistry and Food Technology (IATA), \\ Spanish National Research Council (CSIC), Calle Catedrático Agustín Escardino Benlloch 7, 46980 Paterna, \\ Spain \\ ${ }^{4}$ Department of Experimental and Social Sciences Teaching, Faculty of Teacher Training, Universitat de \\ València (UV). Avda. dels Tarongers, 4, 46022, València, Spain \\ *Corresponding author: A. Ribes-Greus/// aribes@ter.upv.es
}

\begin{abstract}
The influence of the addition of graphene nanoplatelets (GNPs) on the intra/inter - molecular segmental motions of poly(ethylene-co-vinyl alcohol) (EVOH) was assessed by means of dielectric thermal analysis (DETA). The relaxation spectra were studied in terms of the dielectric permittivity $\left(\varepsilon^{\prime}\right)$ and the dielectric loss tangent ( $\tan$ $\delta$ ) at wide ranges of frequency (from $10^{-2}$ to $10^{7} \mathrm{~Hz}$ ) and temperature (from -150 to 140 $\left.{ }^{\circ} \mathrm{C}\right)$. Two relaxation zones were disthinguished. Below the glass transition temperature $\left(T_{g}\right)$, two $\beta$-relaxations were observed, which are characteristic local modes of mobility of the EVOH side groups, and related to the influence of the different surroundings of ethylene or vinyl alcohol units. At higher temperatures, the dielectric $\alpha$-relaxation in the vicinities of the glass transition of $\mathrm{EVOH}$ was determined. The thermal activation of the $\beta$-relaxations was explained by an Arrhenius model, and showed activation energies $\left(E_{a}\right)$ around 55 and $80 \mathrm{~kJ} \cdot \mathrm{mol}^{-1}$. The $\alpha$-relaxation was explained by the Vogel-FulcherTammann-Hesse (VFTH) model. The study of the segmental dynamics showed an increase in the dynamic fragility parameters with the addition of GNPs. The permittivity was increased at preferential concentrations of GNPs. In particular, the addition of GNPs
\end{abstract}


up to $0.5 \mathrm{wt} \%$ increased the dielectric permittivity of the electrospun EVOH/GNPs nanocomposite fibers, specially at low frequencies.

Keywords: poly(ethylene-co-vinyl alcohol) EVOH, graphene nanoplatelets GNPs, polymer nanocomposites, dielectric permittivity, segmental cooperativity, dynamic fragility

\section{Highlights}

- The dielectric performance of electrospun poly(ethylene-co-vinyl alcohol) $(\mathrm{EVOH}) /$ graphene nanoplatelets (GNPs) was determined

- Two sub- $T_{g}$ non-cooperative intramolecular Arrehnius-like $\beta$-relaxations were reported

- A cooperative intermolecular non-Arrhenius-like $\alpha$-relaxation in the vicinities of $T_{g}$ was found

- The addition of GPNs increased the dynamic fragility and decreased the free volume of $\mathrm{EVOH}$

- GNP contents of $0.5 \mathrm{wt} \%$ increased dielectric permittivity of EVOH 


\section{Graphical abstract}

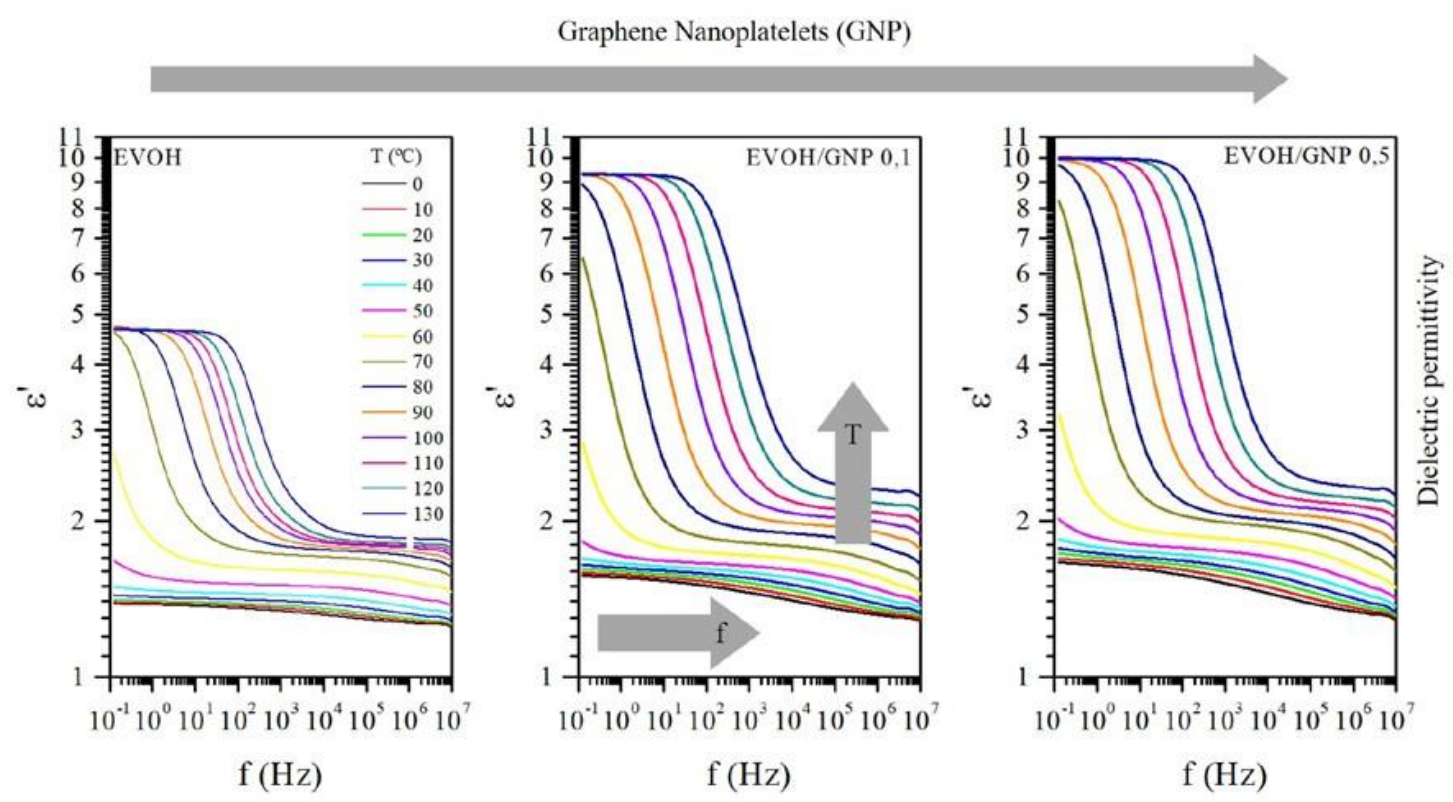




\section{Introduction}

Polymer nanocomposites have recently received a significant attention in both academia and industry for the development of conductive plastics. Since nanofillers are typically below $100 \mathrm{~nm}$ in at least one dimension, they offer ultra-large interfacial area per volume when they are incorporated into a polymer matrix. As a result, the conducting nanofillerreinforced composites exhibit enhanced electrical conductivity over bulk polymers at relatively low proportions of filler, without sacrificing the mechanical behavior and other inherent properties of the polymer matrix. Indeed, to be cost-effective, their predicted electrical improvement must be performed at very low concentrations. To accomplish this, the conducting nanofillers should be dispersed and distributed in such a way to extend and form a continuous network over the whole polymer material. Ideally, all introduced nanofillers should be relatively well interconnected and with low contact resistances among them, to allow the flow of electrical current through the sample [1].

Graphene is a two-dimensional (2D) honeycomb-like monolayer of tightly packed carbon atoms with unique electronic, thermal, and mechanical properties [2]. Graphene nanoplatelets (GNPs) can be incorporated into plastic matrices as multifunctional reinforcing filler [3] without compromising the long-term performance of nanocomposites [4]. The incorporation of GNPs cannot only improve the mechanical and dielectric properties of polymers, but also improve other properties such as thermal conductivity, thermal stability, and gas permeation [5]. In addition, GNPs are capable of dissipating electrostatic charges and shielding devices from electromagnetic radiation in polymer nanocomposites [6]. These properties are related to the dispersion of GNPs, the interfacial adhesion between GNPs and the polymer matrices, or both [7]. Not only the processing conditions and methodology, but also the filler morphology, play a key role in the resulting conductive properties of the polymer nanocomposite [8]. Their dielectric constant can be greatly increased by having numerous nanocapacitors distributed in the polymer matrix, due to the increment of permittivity [9]. Conductive polymer materials are desirable in modern electronics and electric power systems because of their inherent advantages of being easy to process, flexible, and light in weight [10]. They are also relevant in the field of thermoelectric materials, which transform electricity into heat, and viceversa, as alternative energy sources [11]. 
Most studies have been focused on the development of polymer nanocomposites with high permittivity by means of the addition of fillers [12]. Generally, two routes are typically employed to increase the permittivity of polymer composites: i) The use of powders with high permittivity as fillers; ii) The addition of conductive fillers to form a large network of microcapacitors in polymer composites, in which two neighboring conductive fillers serve as electrodes [13]. Graphene has been reported to enhance the dielectric properties of poly(vinylidene fluoride) (PVDF)-based polymers [14], [15], polypropylene (PP) [16] or polyurethanes[17], among others. However, the obtaining of stable and homogeneous dispersions of graphene into polymer matrixes is difficult. It is usually performed by melt blending [18]- which main drawbacks are poor dispersion and risk of thermal degradation-,or solution mixing [19], with the problems related to the used of toxic solvents. In addition, the final shape of polymer products are usually casted films or sheets, which also hiders the well dispersion of fillers in the polymer. As a step forward, the use of electrospinning has been proven to be highly suitable for the incorporation and dispersion of nanoparticles in different ultrathin polymer and biopolymer fibers [20][23]. The resultant electrospun mats composed of composite nanofibers can be thereafter thermally post-processed to produce continuous films [24], which opens up novel applications as new active or bioactive coatings or interlayers, that is, internal layers in a multilayer system, for application in the design of packaging structures [25], [26].

The control of temperature and frequency during the sensing of the permittivity offers a wide range of possibilities for the application of EVOH/GNPs in electrical devices, electrodes, and intelligent packaging, including permanent antistatic films and smart labels. In a previous study [27], the influence of the addition of GNPs into electrospun nanofibers of poly(ethylene-co-vinyl alcohol) (EVOH), was assessed in terms of scanning electron microscopy (SEM), transmission electron microscopy (TEM), wide-angle X-ray scattering (WAXS), Raman imaging and differential scanning calorimetry (DSC). It was shown that the GNPs were well dispersed between 0.1 and $2 \mathrm{wt} \%$. In addition, the GNPs acted as anti-nucleant agent for the EVOH macromolecules, particularly at low contents, due to its improved dispersion and high interaction with the polymer matrix. In addition, preliminary studies by means of dielectric thermal analysis by frequency sweeps at isothermal conditions of $25{ }^{\circ} \mathrm{C}$ were performed. The incorporation of low contents of nanofiller sharply increased the conductivity of the electrospun EVOH fibers, up to a 
content of 0.5 wt.- $\%$ GNPs, with a percolation threshold relatively close to 0.1 wt.- $\%$ GNPs.

Based on the previous results, it is therefore relevant to continue with the studies of dielectric properties of the EVOH/GNPs nanocomposites. In this work, the macromolecular movements related to the dipole orientation in EVOH/GNPs nanofibers were deeply studied by dielectric thermal analysis (DETA), extending the analysis to the frequency range from $10^{-2}$ to $10^{7} \mathrm{~Hz}$, and the temperature range from -150 to $140{ }^{\circ} \mathrm{C}$. DETA is a very useful tool to study the impact on the dielectric permitivitty and segmental cooperativity of polymers [28]-[36] as the response to an electrical perturbation field over a wide frequency range at different temperatures, which modifications are not completely perceptible by other macroscopic techniques. Therefore, the aim of this study was to analyse the influence of the addition of GNPs to EVOH nanofibers in terms of dielectric permittivity and segmental cooperativity. 


\section{Experimental procedure and calculation methods}

\subsection{Materials and preparation of electrospun mats}

EVOH copolymer with 32-mol\% ethylene, i.e., EVOH32, traded as SoarnoL ${ }^{\mathrm{TM}}$ DC3212B, was supplied by Nippon Gohsei (Ogaki, Japan). Graphite oxide (GO) was produced in-house by the modified Hummer's method using graphite powder and, afterwards, it was reduced to graphene using hydrazine hydrate [27].

Electrospun EVOH/GNPs nanofibers were obtained by electrospinning using a Fluidnatek ${ }^{\circledR}$ LE500 pilot line from Bioinicia S.L. (Valencia, Spain) with a voltage of 10 $\mathrm{kV}$, a tip-to-collector distance of $15 \mathrm{~cm}$, and a flow-rate of $0.2 \mathrm{~mL} / \mathrm{h}$. All samples were electrospun in a controlled environmental chamber at $29^{\circ} \mathrm{C}$ and $30 \%$ relative humidity (RH). The electrospun mats were collected on a metallic collector and then stored under dry conditions. Following compositions were prepared: Neat electrospun EVOH nanofibers (control) and EVOH with GNPs contents of 0.1, 0.5, 1, and 2 wt.\%. Further details about the electrospinning process and conditions can be found in the previous research work [27]. Concerning the morphology, neat EVOH fibres presented a fibrillary morphology, free of beaded regions, with a mean diameter of $\sim 675 \mathrm{~nm}$. An increasing addition of GNPs led to a reduction of average diameter from $425 \mathrm{~nm}$ from those filled with $0.1 \mathrm{wt} \%$ towards below $100 \mathrm{~nm}$ for those filled with $2 \mathrm{wt} \%$, due to the electrical conductivity of the electrospun solution. Macroscopically, the samples were workable films with $\sim 200 \mu \mathrm{m}$ thickness. Morphological (SEM) and Raman analyses revealed that the best GNPs dispersion was obtained for the electrospun EVOH nanomats containing 0.5 wt.-\% GNPs. However, GNPs were likely to increasingly agglomerate as their concentration was increased. At GNPs loads higher than $2 \mathrm{wt} \%$, beads started to be prominent in the morphologies.

\subsection{Dielectric characterisation}

The dielectric spectra (DS) of the EVOH-GNPs nanocomposites were obtained using an alpha mainframe frequency analyser in conjunction with an active cell (Concept 40, Novocontrol Technologies BmgH \& Co. Kc, Hundsangen, Germany). The relative response was measured in the frequency range $f=10^{-2}-10^{7} \mathrm{~Hz}$, at temperatures of -150 
${ }^{\circ} \mathrm{C}$ to $140{ }^{\circ} \mathrm{C}$, controlled by the Quatro system (Novocontrol Technologies, Germany). The spectra were obtained under isothermal conditions by increasing steps of $10{ }^{\circ} \mathrm{C}$. The sample electrode assembly (SEA) consisted of two stainless steel electrodes filled with the polymer, along with a $132 \mu \mathrm{m}$ thick Teflon film, as conductive-blocking layer [37][39]. The Teflon film between one of the electrodes and the sample allows determining relaxations that are normally hidden by the usually large contribution of conductivity and polarisation effects at high temperatures and low frequencies. The position and width of the relaxations are not affected by conductivity, which permits a detailed characterisation. The diameters of the electrodes were $20 \mathrm{~mm}$ and the thickness was kept around $300 \mu \mathrm{m}$.

\subsection{Analysis of segmental cooperativity}

In order to assess the influence of GNPs on the electrospun EVOH nanofibers, the dielectric curves were deconvoluted by means of the Charlesworth method [40] using Havriliak-Negami (HN) fitting functions (Eq. 1) [41], [42].

$$
\varepsilon^{*}(w)-\varepsilon_{\infty}=\sum_{k} \operatorname{Im}\left[\frac{\Delta \varepsilon}{\left\{1+\left(i w \tau_{H N k}\right)^{\alpha_{k}}\right\}^{\beta_{k}}}\right]
$$

, where $\alpha_{k}$ and $\beta_{k}$ are parameters correspond to the width and asymmetry of the relaxation time distributions, respectively; $\tau_{H N}$ is the Havriliak-Negami relaxation time, and $k$ represents the number of the individual HN contributions, which can vary from $k=1$ to 3 , depending on the complexity of the $\varepsilon$ "' curve at any given temperature.

The thermal activation of the dielectric phenomena was characterized by means of Arrhenius maps (i.e $\log f_{\max } \mathrm{vs} . \mathrm{T}^{-1}$ ), being $\mathrm{f}_{\max }=\tau_{\max }{ }^{-1}$ the frequency related to the most probable relaxation time, determined from the $\mathrm{HN}$ deconvoluted curves at a certain isotherm $\mathrm{T}(\mathrm{Eq} 2)$. 


$$
\tau_{\max }=\tau_{H N}\left[\frac{\sin \left(\frac{\pi\left(\alpha_{N H}\right) \beta_{N H}}{2\left(\beta_{N H}+1\right)}\right)}{\sin \left(\frac{\pi\left(\alpha_{N H}\right)}{2\left(\beta_{N H}+1\right)}\right)}\right]^{1 / \alpha_{N H}} \text { (Eq. 2) }
$$

Furthermore, the temperature dependence of the relaxation times was analysed by means of the Eyring equation (Eq.3) [43], [44].

$$
E a=R T^{\prime}\left[1+\ln (k / 2 p h)+\ln T^{\prime}\right]=R T^{\prime}\left[22.92+\ln T^{\prime}\right] \quad \text { (Eq. 3) }
$$

, where $T^{\prime}$ is the temperature at which the frequency of the corresponding relaxation is 1 Hz. Eq.3 represents an universal line in coordinates $E_{a}$ vs T' in which the relaxations with zero-entropy should fall [45]. This evaluation permits to discriminate cooperative and non-cooperative segmental motions since cooperative relaxations would show $\left\{\mathrm{Ea}, \mathrm{T}^{\prime}\right\}$ coordinates close to the zero entropy line, whereas non-cooperative relaxations will show higher $\mathrm{Ea}$, and therefore $\left\{\mathrm{Ea}, \mathrm{T}^{\prime}\right\}$ coordinates would appear far above the zero-entropy line.

\subsection{Assessment of thermal activation of dielectric relaxations}

Both cooperative and non-cooperative intramolecular relaxations can be mathematically described in terms of Arrhenius-like behaviors (Eq. 4):

$$
f_{\max }=f_{0} \exp \left(\frac{-E a}{R \cdot T}\right) \quad \text { (Eq.4) }
$$

, where $f$ and $\mathrm{T}$ are the linear frequency and temperature of the DETA tests, $E_{a}$ is the apparent activation energy, $f_{0}$ is a pre-exponential term, and $\mathrm{R}$ is the ideal gas constant $\left(8.31 \mathrm{~J} \cdot \mathrm{mol}^{-1} \cdot \mathrm{K}^{-1}\right)$.

The dependence of the relaxation times with the temperature for segmental movements at temperatures higher than the glass transition is usually governed by a Vogel-FulcherTammann-Hesse (VFTH) [46], [47] (Eq. 5): 


$$
f_{\max }=f_{0} \cdot \exp \left(-\frac{B}{T-T_{V F T H}}\right)=f_{0} \cdot \exp \left(-\frac{D \cdot T_{V F T H}}{T-T_{V F T H}}\right)
$$

(Eq. 5)

, where $f_{0}$ is a pre-exponential term, $B(K)$ is an activation parameter, and $T_{V F T H}(\mathrm{~K})$ is the VFTH temperature, which are positive temperature-independent empirical parameters, specific to the material. It is common to rewrite the parameter $B$ into $B=D \cdot T_{V F T H}$, where $D$ is a non-dimensional factor termed as fragility or strength parameter. Additionally, the so-called fragility index $m$ permits an assessment of the deviation of $\tau(T)$ from the Arrhenius-like behavior of polymer relaxations that can be obtained by $E q$. 6 . The influence of the structure of polymers on their fragility can be found elsewhere [48]. The free volume coefficient $\Phi$ was calculated according to $E q 7$, after comparison of the VFTH model with the Doolitle expression [49], [50]. According to Fujimori and Oguni [51], the non-Arrhenius behavior of the $\alpha$-relaxation could also be interpreted as caused by changes in the activation energy. The value of this parameter can be calculated at a specific temperature, such as the glass transition temperature $\left(T_{g}\right)$, which is the commonly used reference $(E q 8)$.

$$
\begin{aligned}
& m=\left.\frac{\partial \log (\tau)}{\partial\left(\frac{T_{G}}{T}\right)}\right|_{T_{g}}=\frac{B \cdot T_{g}}{\ln (10) \cdot\left(T_{g}-T_{V F T H}\right)^{2}} \\
& \left.\phi\right|_{T g}=\frac{\left(T_{g}-T_{V F T H}\right)}{B} \\
& E a_{T g}=R \cdot \frac{\partial \ln \tau}{\partial(1 / T)}=\frac{R \cdot B}{\left(1-\frac{T_{\text {VFTH }}}{T_{g}}\right)^{2}}
\end{aligned}
$$




\section{Results and Discussion}

\subsection{Phenomenological description of dielectric relaxations}

The analysis was essentially conducted through the monitoring of loss tangent $(\tan \delta)$ and the complex dielectric permeability $\varepsilon^{*}=\varepsilon^{\prime}-i \varepsilon^{\prime}$, taking into account the real $\left(\varepsilon^{\prime}\right)$ and imaginary $(\varepsilon ")$ parts. The three-dimensional dielectric spectrum of the electrospun $\mathrm{EVOH}$ and $\mathrm{EVOH} / \mathrm{GNP}$ nanofibers in terms of $\tan \delta$ along a large range of frequency and temperature are shown in Figure 1. For a deep identification of the dielectric relaxations, the isochronal loss tan $\delta$ curves as a response of temperature are given in Figure 2. As expected, higher frequencies shifted the dielectric signals to higher temperatures. The spectra are relatively similar to those shown for neat electrospun EVOH nanofibers by means of dynamic mechanical thermal analysis (DMTA) [52] or DETA [53]. DETA offers a frequency range wider than DMTA, which permits a deep discussion on macromolecular relaxations involved in the polarisation movements.

The dielectric spectra of the EVOH/GNP nanocomposites exhibited, in an increasing temperature order, two sub- $T_{g} \beta$-relaxations in the glassy state and one $\alpha$-relaxation. At low temperatures, the two sub- $T_{g} \beta$-relaxations in the glassy state appeared for all studied frequencies for both electropun neat EVOH and EVOH/GNP nanofibers, which might be ascribed to local modes of mobility of the side groups of EVOH. The apparition of two close transitions may be related to the surroundings of ethylene and vynil alcohol groups of the EVOH chains, which differences in its polarisable groups split the dielectric response into two close relaxations. Figure 3 shows an example of devonvolution of the $\beta$ relaxation zone by means of 2 Havriliak-Negami functions. The detection of the presence of $2 \beta$-relaxations at low temperatures and low $\tan \delta$ was strengthened by further analysis of the thermal activation of these relaxations, as it is shown in the following section.

At higher temperatures, the dielectric $\alpha$-relaxation corresponding to the glass-rubber transition of EVOH was found. The significant increment of the loss factor, in contrast to the values given by the $\beta$-relaxations, resulted from the inability of the polarization process of the macromolecules to follow the rate of change of the oscillating applied electric field. Actually, when the rate of electric field oscillates well faster than the macromolecular relaxation time, the polarization cannot follow the oscillating frequency 
resulting in energy absorption and is dissipated as heat [32]. The $\alpha$-relaxation appeared between $10^{1}$ and $10^{3} \mathrm{~Hz}$, at $\mathrm{T}>50^{\circ} \mathrm{C}$ for $\mathrm{EVOH}$, while it was observable between $10^{-1}$ and $10^{3} \mathrm{~Hz}$ for $\mathrm{EVOH} / \mathrm{GNP}$ nanofibers at similar temperature, which indicated positive interaction through hydrogen bonding between EVOH and graphene. The aforementioned assignation of segmental movements is discussed in the following sections, in terms of cooperativity and energies associated to the macromolecular motions.

\subsection{Effect of the addition of GNPs on the dielectric permittivity $\varepsilon$ ' of EVOH}

The plots corresponding to the frequency-response of the dielectric permittivity $\varepsilon$ ' are shown in Figure 4 for the electrospun neat EVOH and EVOH/GNP nanofibers, at low and high temperature ranges. Different effects can be drawn depending on the focus of analysis, i.e, influence of temperature, frequency, and addition of GNPs.

On the one hand, at a certain frequency, for all the analysed frequency range, the higher the temperature was, the higher the dielectric permittivity $\varepsilon$ ' was registered. As expected, since temperature increases molecular mobility, therefore it eases the adaptation of macromolecules to the polarization of the DETA electric field [32], i.e. thermal agitation facilitates the orientation of polar groups.

On the other hand, at a certain temperature, at low frequencies, the dielectric permittivity $\varepsilon$ 'attained higher values for all EVOH/GNP nanofibers cases, which afterwards diminished rapidly with increasing frequency. In the low frequency region, the alternation of the electric field is slow, thus providing sufficient time to permanent and induced dipoles to align themselves according to the applied field, leading to enhanced polarization [54]. Afterwards, a significant difference between the low- $f$ and high- $f$ dielectric permittivity $\varepsilon$ ' remarked the identification of the glass transition temperature $T_{g}$ between 50 and $60{ }^{\circ} \mathrm{C}$. This remarkable reduction of permitivity is due to the initiation of micro-Brownian motions of the macromolecular chains from the frozen state during the increase of temperature. The results of $T g$ are in close agreement with those previously reported by means of differential scanning calorimetry (DSC) and dynamicalmechanical-thermal-analysis (DMTA), with no significant differences among materials [55]-[57]. As previously shown in Figure 2, the $T_{g}$ could be also observed at the temperature corresponding to the maximum of the $\tan \delta$ peak in which the first remarkable 
loss of energy was registered, which occured at $10^{1} \mathrm{~Hz}$ for the neat EVOH nanomats and $10^{-1} \mathrm{~Hz}$ for EVOH/GNP nanomats.

Finally, concerning the addition of GNPs, the DETA analysis evidenced an increase of the dielectric permittivity $\varepsilon^{\prime}$, up to a $0.5 \%$ wt of graphene content in the electrospun $\mathrm{EVOH}$, specially at low frequencies. This effect is ascribed to the contribution of interfacial polarisation to net polarization present in the system. Due to the different relaxation times of EVOH matrix and the GNPs, charge carriers can be generated by surface polarization and between both components. The interfacial polarization occurred at low frequency, while decreased at higher frequencies, when the relaxation times of the EVOH matrix led the whole polarisation process [58]. In contrast, electrospun EVOH filled with 1 and 2 wt\% of GNPs reduced the dielectric permittivity $\varepsilon$ ' to similar values to those observed for neat EVOH. This reduction can be ascribed to the formation of aggregates of GNPs in the EVOH matrix [27], which might difficult percolation and hindrance dipole transfer, as also reported for EVOH/GNPs casted films [54]. This effect could also be addressed to hydrogen bonding at the interface of EVOH and GNPs, which might impair the dipole transfer [59]. A similar behaviour was also observed in the preliminary study of these electrospun mats for the electric conductivity, in which the highest value was also attained for GNPs contents of $0.5 \%$ wt [27].

\subsection{Identification of cooperative behavior of relaxations}

The relationship between relaxation times and temperature was displayed in Arrhenius maps, which plot the $\log (f) v s . T^{-1}$, as shown in Figure 5a, for the electrospun neat EVOH and its EVOH/GNP nanomats. Two different behaviors can be distinguished, regarding the thermal activation of the dielectric relaxations. On the one hand, at low temperatures, two almost parallel relaxations were related to the non-cooperative movements of the intramolecular group motions, which displayed a linear relationship between $\log (f)$ and $T^{-1}$, according to the Arrhenius equation (Eq. 4). On the other hand, a curved relationship between $\log (f)$ and $T^{-1}$ at higher temperatures can be explained in terms of a VFTH equation (Eq. 5), which is related to intermolecular cooperative movements of the copolymer backbone.

In order to ensure the classification of the dielectric relaxations in terms of cooperative or non-cooperative movements, the Eyring plot in Figure $\mathbf{5 b}$ shows the relationship 
between the $E_{a}$ of each identified relaxation and $T$,', which is the temperature that corresponds to the maximum of the $\tan \delta$ at $1 \mathrm{~Hz},(E q .3)$. On the one hand, the $\left\{E a, T^{\prime}\right\}$ coordinates for the $\beta$-relaxations were depicted close to the zero-entropy line and, therefore, could be ascribed to non-cooperative intramolecular relaxations. On the other hand, the $\left\{E a, T^{\prime}\right\}$ coordinates for the $\alpha$-relaxation appeared far from the zero-entropy line and were thus related to intermolecular cooperative relaxations. This effect was more prominent with the addition of GNPs, showing the highest $E a$ for the EVOH nanofibers filled with $0.5 \%$ wt of GNPs.

\subsection{Analysis of non-cooperative intramolecular relaxations in the glassy state}

The dielectric processes appearing in the glassy state are labelled as $\beta_{I}$ and $\beta_{I I}$ in the increasing order of temperatures. Table 1 gathers the results of the Arrhenius fitting ( $E q$. 4) to $\beta_{I}$ and $\beta_{I I}$ intramolecular non-cooperative relaxations. At low temperatures, below the $T_{g}$, the macromolecular mobility was severely restrictive and therefore relatively low activation energies $E a$ were expected. The $E_{a}$ values obtained for the $\beta_{I}$-relaxation $(\sim 47-$ $\left.58 \mathrm{~kJ} \cdot \mathrm{mol}^{-1}\right)$ and $\beta_{I I}$-relaxation $\left(\sim 76-89 \mathrm{~kJ} \cdot \mathrm{mol}^{-1}\right)$ were close to those reported for other EVOH co-polymers [52]. Several cases of molecular motions are proposed as origin of these relaxations processes, as it would depend on the relative relevance of the movements of the ethylene and vinyl alcohol segments within the EVOH structure. On the one hand, the $\beta$-relaxation has been reported for branched polyethylenes at temperatures around $-20{ }^{\circ} \mathrm{C}$, being ascribed to ethylene motions of chain units in the interfacial region. These is in agreement with previous reports by Madelkern's group, which analysed the macromolecular relaxations of polyethylene and polyethylene-based copolymers by means of DMTA and DETA [55], [60]. Accordingly, both the temperature location of the $\beta_{I}$ peak and the smaller value of the $E a$ suggested a mechanism for the EVOH nanocomposites similar to that reported for polyethylene. On the other hand, the $\beta$ - relaxation mechanism in poly(vinyl alcohol) $(\mathrm{PVOH})$ has been attributed in literature either to hindered rotations of water molecules bound by hydrogen bonds to the polymer chains [61], [62] or to local rotation and torsion movements of the vynil alcohol segments and non-bonded $-\mathrm{OH}$ groups [56]. Both the location of the peak temperature of the $\beta_{I I}$ relaxation and the higher value of the apparent activation energy suggested a mechanism for the EVOH nanocomposites similar to that reported for PVOH. Since the samples were 
carefully dried, the rotation movements of the non-bonded -OH groups of the vynil alcohol segments were considered more likely to experience the $\beta_{I I}$-relaxation.

The addition of GNPs to EVOH slightly modified the $E_{a}$ values of both $\beta$-relaxations. The small obtained modifications can be related to the competitive effect between effective dipole charge transfer and the hindrance of movements by hydrogen bonding with the GNPs.

Table 1. Results of Arrhenius fitting to $\beta_{I}$ and $\beta_{I I}$ intramolecular non-cooperative relaxations of the electrospun poly(ethylene-co-vinyl alcohol) (EVOH) and EVOH/ graphene nanoplatelets (GNPs) in terms of the the apparent activation energy $\left(E_{a}\right)$ and the pre-exponential term $\left(\log f_{0}\right)$

\begin{tabular}{c|ccc|ccc} 
& \multicolumn{3}{|c|}{$\beta_{\text {I-relaxation }}$} & \multicolumn{3}{c}{$\beta_{\text {II-relaxation }}$} \\
\hline & $E_{a}\left(\mathbf{k J} \cdot \mathbf{m o l}^{-1}\right)$ & $\log f_{0}(\mathbf{H z})$ & $\mathbf{R}^{2}$ & $\left.E_{a}(\mathbf{k J} \cdot \mathbf{m o l})^{-1}\right)$ & $\log f_{0}(\mathbf{H z})$ & $\mathbf{R}^{2}$ \\
\hline EVOH & $48.12 \pm 0.88$ & $14.62 \pm 0.17$ & 0.997 & $89.03 \pm 5.27$ & $19.73 \pm 1.10$ & 0.986 \\
EVOH/GNP 0.1 & $46.94 \pm 1.37$ & $14.47 \pm 0.25$ & 0.992 & $75.32 \pm 1.10$ & $17.63 \pm 0.22$ & 0.999 \\
EVOH/GNP 0.5 & $54.54 \pm 2.20$ & $15.13 \pm 0.45$ & 0.982 & $76.01 \pm 7.80$ & $18.09 \pm 1.66$ & 0.968 \\
EVOH/GNP 1 & $61.98 \pm 2.34$ & $17.06 \pm 0.46$ & 0.985 & $76.57 \pm 3.08$ & $17.46 \pm 0.61$ & 0.990 \\
EVOH/GNP 2 & $58.27 \pm 0.97$ & $16.30 \pm 0.18$ & 0.997 & $80.85 \pm 3.84$ & $18.03 \pm 0.81$ & 0.989
\end{tabular}

\subsection{Analysis of cooperative intermolecular relaxations in the rubbery state}

At high temperatures, a very high intensity zone constituted the $\alpha$-relaxation, which was related to the cooperative intermolecular movements in the vicinities of the glass transition. When a polymer evolves from the glassy to the rubbery state, structural arrangements associated with micro-Brownian motions are registered. The mean relaxation time, which characterise the cooperative motions of polymer segments of the main backbone, changed many orders of magnitude. Therefore, the dielectric relaxations could be explained by means of the empirical Vogel-Fulcher-Tamman-Hesse VFTH equation (Eq. 5). The confidence $\mathrm{R}^{2}$-values of the fitting were $0.987,0.988,0.996,0.989$, 
and 0.990 for electrospun nanomats of neat EVOH, and EVOH/GNP 0.1, EVOH/GNP 0.5, EVOH/GNP 1, and EVOH/GNP 2, respectively.

The segmental motions were assessed in terms of the evolution of dynamic fragility indicators with the increment of GNPs in the electrospun EVOH nanomats. The dynamic fragility parameter $D$, activation parameter $B$, dynamic fragility index $m$, free volume coefficient $\Phi$, and activation energy at the glass transition $E a_{T g}$ were calculated as shown above, and plotted in Figure 6 as a function of the GNPs content in EVOH. The $T_{g}$ obtained by differential scanning calorimetry [27] was used as reference temperature for calculations. The error bars ranged between 2 and $6 \%$, and were omitted for the sake of clarity.

A qualitative distinction between strong and fragile dynamic behaviors can be drawn from the analysis of the parameters $D$ and $m$. On the one hand, $D$ is related to the topology of the theoretical potential energy surface of the system, where fragile systems $(D \leq 6)$ present high density of minimum energy, contrarily to strong systems $(D \geq 15)$ which present lower density. On the other hand, $m$ varies between two limiting values of 16 and $\geq 200$ for strong and fragile glass-formers, respectively [63]. Electropun EVOH and EVOH/GNP nanomats showed a strong glass-former behavior regardless the proportion of GNPs in the formulation ( $m \sim 40-55)$. The values of the dynamic fragility parameters $D$ and $B$ resulted in the same order of magnitude than those reported by Tantis et al. for $\mathrm{EVOH} /$ graphite casted films [54].

The addition of GNPs increased $D$ and subsequently $B$, which are associated to the increment of activation energy to overcome the glass transition $\left(E a_{T g}\right)$. These results could be explained in terms of the free volume at the vicinities of the glass transition, which decreased with the higher loads of GNPs. Since a thermal treatment of nanocomposites was not applied, degradation during preparation was not expected. Therefore, the variation of dynamic fragility indicators was related to the interactions of GNPs with the EVOH nanofibers. The decrease of free volume might induce a stiffening effect that hindered the macromolecular movements and thus reduced the dynamic fragility parameters. All effects were more relevant after the addition of GNPs to the EVOH matrix. However, for contents higher than $0.1 \mathrm{wt} \%$ of GNPs, the tendency of the indicators was maintained under a certain order of magnitude, which trend seems to reach 
a plateau. As it can be seen in the figure, the main difference can be observed from non-loaded EVOH towards loaded EVOH/GNPs nanomats. At the range of content of the GNPs of the present study, big differences were not expected among EVO/GNPs. Since for concentrations higher than $2 \mathrm{wt} \%$ the dispersion loses hetereogeneity, a major evolution of the thermal activation parameters was not foreseen at an operational level of the EVOH/GNP nanomats. 


\section{Conclusions}

The dielectric permittivity of the nanocomposites based on EVOH filled with GNPs can be modified by the fine-tuning of their composition, in terms of nanofillers amount of polymer matrix. In this sense, low contents of nanofillers were sufficient to induce an increase in permittivity.

The addition of GNPs did not hinder the macromolecular segmental movements. Below the $T_{g}$ of the EVOH/GNPs nanocomposites, two non-cooperative intramolecular $\beta$ relaxations were found and explained in terms of the Arrhenius behavior. At high frequencies, the $\beta_{I}$ relaxation was ascribed to the local movements related to the ethylene part of the $\mathrm{EVOH}$, while at low frequencies the $\beta_{I I}$ relaxation was ascribed to those of the vynil-alcohol segments. Additionally, in the vicinities of the glass transition of the EVOH/GNPs nanocomposites, a cooperative intermolecular $\alpha$-relaxation was explained in terms of Vogel-Fulcher-Tamman-Hesse behavior, which was ascribed to the microbrownian motions related to the structural arrangements from the glassy to the rubbery state. The addition of GNPs increased the dynamic fragility of the nanocomposites, and more energy was necessary to overcome the glass transition. This effect was related to a reduction of the free volumein the vicinities of the glass transition, subsequently hindering the macromolecular movements. Finally, the addition of GNPs up to a $0.5 \mathrm{wt} \%$ increased the dielectric permittivity of the electrospun nanofibers, specially at low frequencies.

\section{Acknowledgments}

The authors would like to thank the European Regional Development Funds (ERDF) and the Spanish Ministry of Science, Innovation, and Universities (MICIU) for the concession of the Research Projects ENE2017-86711-C3-1-R and RTI2018-097249-B-C21. The UPV authors would also like to thank the Chilean Economic Development Agency (CORFO) the economic support in the frame of the project 13CEI2-21839. This research was also funded by the EU H2020 YPACK (773872) and USABLE (BBI-JTI-2018836884) projects. Torres-Giner is a recipient of a Juan de la Cierva-Incorporación contract (IJCI-2016-29675) from MICIU. Universitat Politècnica de València is acknowledged for the post-doctoral aid for R. Teruel-Juanes, under the PAID-10-19 SUB.1 SP20190049 scholarship. 
- 20 - 


\section{References}

[1] S. Torres-Giner, "Preparation of conductive carbon black-filled polymer nanocomposites via melt compounding.," in Conductive Materials and Composites, Nova Science Publishers, 2016, pp. 117-164.

[2] A. K. Geim, "Graphene: Status and Prospects," Science (80-. )., vol. 324, no. 5934, pp. 1530 LP - 1534, Jun. 2009.

[3] H. Kim, A. A. Abdala, and C. W. Macosko, "Graphene/Polymer Nanocomposites," Macromolecules, vol. 43, no. 16, pp. 6515-6530, Aug. 2010.

[4] J. D. Badia, O. Gil-Castell, and A. Ribes-Greus, "Long-term properties and endof-life of polymers from renewable resources," Polym. Degrad. Stab., Mar. 2017.

[5] H. Bai, C. Li, and G. Shi, "Functional composite materials based on chemically converted graphene.," Adv. Mater., vol. 23, no. 9, pp. 1089-115, Mar. 2011.

[6] J. Li and J.-K. Kim, "Percolation threshold of conducting polymer composites containing 3D randomly distributed graphite nanoplatelets," Compos. Sci. Technol., vol. 67, no. 10, pp. 2114-2120, Aug. 2007.

[7] T. Kuilla, S. Bhadra, D. Yao, N. H. Kim, S. Bose, and J. H. Lee, "Recent advances in graphene based polymer composites," Prog. Polym. Sci., vol. 35, no. 11, pp. 1350-1375, 2010.

[8] S. Torres-Giner, A. Chiva-Flor, and J. L. Feijoo, "Injection-molded parts of polypropylene/multi-wall carbon nanotubes composites with an electrically conductive tridimensional network," Polym. Compos., vol. 37, no. 2, pp. 488496, 2016.

[9] K. Ahmad, W. Pan, and S.-L. Shi, "Electrical conductivity and dielectric properties of multiwalled carbon nanotube and alumina composites," Appl. Phys. Lett., vol. 89, no. 13, p. 133122, Sep. 2006.

[10] C. Wu, X. Huang, L. Xie, X. Wu, J. Yu, and P. Jiang, "Morphology-controllable graphene-TiO2 nanorod hybrid nanostructures for polymer composites with high dielectric performance,” J. Mater. Chem., vol. 21, no. 44, pp. 17729-17736, 
[11] C. Gao and G. Chen, "Conducting polymer/carbon particle thermoelectric composites: Emerging green energy materials," Compos. Sci. Technol., vol. 124, pp. 52-70, Mar. 2016.

[12] B. Chu et al., "A Dielectric Polymer with High Electric Energy Density and Fast Discharge Speed,” Science (80-. )., vol. 313, no. 5785, pp. 334 LP - 336, Jul. 2006.

[13] V. Rubentheren, T. A. Ward, C. Y. Chee, and C. K. Tang, "Processing and analysis of chitosan nanocomposites reinforced with chitin whiskers and tannic acid as a crosslinker," Carbohydr. Polym., vol. 115, pp. 379-387, 2015.

[14] P. Fan, L. Wang, J. Yang, F. Chen, and M. Zhong, “Graphene/poly(vinylidene fluoride) composites with high dielectric constant and low percolation threshold.," Nanotechnology, vol. 23, no. 36, p. 365702, Sep. 2012.

[15] D. Wang, Y. Bao, J. W. Zha, J. Zhao, Z. M. Dang, and G. H. Hu, "Improved dielectric properties of nanocomposites based on poly(vinylidene fluoride) and poly(vinyl alcohol)-functionalized graphene," ACS Appl. Mater. Interfaces, vol. 4, no. 11, pp. 6273-6279, 2012.

[16] D. Wang, X. Zhang, J.-W. Zha, J. Zhao, Z.-M. Dang, and G.-H. Hu, "Dielectric properties of reduced graphene oxide/polypropylene composites with ultralow percolation threshold," Polymer (Guildf)., vol. 54, no. 7, pp. 1916-1922, 2013.

[17] C. Wu et al., "Hyperbranched-polymer functionalization of graphene sheets for enhanced mechanical and dielectric properties of polyurethane composites," $J$. Mater. Chem., vol. 22, no. 14, pp. 7010-7019, 2012.

[18] L. Cabedo, M. P. Villanueva, J. M. Lagarón, and E. Giménez, “Development and characterization of unmodified kaolinite/EVOH nanocomposites by melt compounding," Appl. Clay Sci., vol. 135, pp. 300-306, 2017.

[19] I. Topolniak, J.-L. Gardette, and S. Therias, "Influence of zeolite nanoparticles on photostability of ethylene vinyl alcohol copolymer (EVOH)," Polym. Degrad. Stab., vol. 121, pp. 137-148, 2015. 
[20] S. Torres-Giner and J. M. Lagaron, “Zein-based ultrathin fibers containing ceramic nanofillers obtained by electrospinning. I. Morphology and thermal properties," J. Appl. Polym. Sci., vol. 118, no. 2, pp. 778-789, 2010.

[21] A. Cherpinski, M. Gozutok, H. Sasmazel, S. Torres-Giner, and J. Lagaron, "Electrospun oxygen scavenging films of poly (3-hydroxybutyrate) containing palladium nanoparticles for active packaging applications," Nanomaterials, vol. 8, no. 7, p. 469, 2018.

[22] O. Gil-Castell, J. D. D. Badia, and A. Ribes-Greus, "Tailored electrospun nanofibrous polycaprolactone/gelatin scaffolds into an acid hydrolytic solvent system,” Eur. Polym. J., vol. 101, pp. 273-281, Apr. 2018.

[23] O. Gil-Castell, J. D. Badia, I. Ontoria-Oviedo, D. Castellano, P. Sepúlveda, and A. Ribes-Greus, "Polycaprolactone/gelatin-based scaffolds with tailored performance: in vitro and in vivo validation," Mater. Sci. Eng. C, vol. 107, p. 110296, 2020.

[24] A. Cherpinski, S. Torres-Giner, L. Cabedo, and J. M. Lagaron, "Post-processing optimization of electrospun submicron poly (3-hydroxybutyrate) fibers to obtain continuous films of interest in food packaging applications," Food Addit.

Contam. Part A, vol. 34, no. 10, pp. 1817-1830, 2017.

[25] S. Torres-Giner, R. Pérez-Masiá, and J. M. Lagaron, “A review on electrospun polymer nanostructures as advanced bioactive platforms," Polym. Eng. Sci., vol. 56, no. 5, pp. 500-527, 2016.

[26] Y. Echegoyen, M. J. Fabra, J. L. Castro-Mayorga, A. Cherpinski, and J. M. Lagaron, "High throughput electro-hydrodynamic processing in food encapsulation and food packaging applications," Trends Food Sci. Technol., vol. 60, pp. 71-79, 2017.

[27] S. Torres-Giner, Y. Echegoyen, R. Teruel-Juanes, J. Badia, A. Ribes-Greus, and J. Lagaron, "Electrospun Poly (ethylene-co-vinyl alcohol)/Graphene Nanoplatelets Composites of Interest in Intelligent Food Packaging Applications," Nanomaterials, vol. 8, no. 10, p. 745, 2018. 
[28] J. D. Badia, L. Monreal, V. Sáenz De Juano-Arbona, and A. Ribes-Greus, "Dielectric spectroscopy of recycled polylactide," Polym. Degrad. Stab., 2014.

[29] S. A. Cruz and M. Zanin, "Dielectric strength of the blends of virgin and recycled HDPE,” J. Appl. Polym. Sci., vol. 91, no. 3, pp. 1730-1735, 2004.

[30] W. Zheng and S.-C. Wong, "Electrical conductivity and dielectric properties of PMMA/expanded graphite composites," Compos. Sci. Technol., vol. 63, no. 2, pp. 225-235, 2003.

[31] S. Singha and M. J. Thomas, "Dielectric properties of epoxy nanocomposites," IEEE Trans. Dielectr. Electr. Insul., vol. 15, no. 1, pp. 12-23, 2008.

[32] E. Riande, R. Diaz-Calleja, M. Prolongo, R. Masegosa, and C. Salom, Polymer viscoelasticity: stress and strain in practice. CRC Press, 1999.

[33] J. D. Badia, R. Teruel-Juanes, C. Acebo, O. Gil-Castell, A. Serra, and A. RibesGreus, "Dielectric spectroscopy of novel thiol-ene/epoxy thermosets obtained from allyl-modified hyperbranched poly(ethyleneimine) and diglycidylether of bisphenol A,” Eur. Polym. J., vol. 113, no. November 2018, pp. 98-106, 2019.

[34] A. M. Salaberría et al., "Influence of chitin nanocrystals on the dielectric behaviour and conductivity of chitosan-based bionanocomposites," Compos. Sci. Technol., vol. 167, no. February, pp. 323-330, 2018.

[35] O. Gil-Castell et al., "Crosslinked chitosan/poly(vinyl alcohol)-based polyelectrolytes for proton exchange membranes," React. Funct. Polym., vol. 142, pp. 213-222, 2019.

[36] O. Gil-Castell, D. Galindo-Alfaro, S. Sánchez-Ballester, R. Teruel-Juanes, J. D. Badia, and A. Ribes-Greus, "Crosslinked sulfonated poly(Vinyl alcohol)/graphene oxide electrospun nanofibers as polyelectrolytes," Nanomaterials, vol. 9, no. 3, pp. 1-20, 2019.

[37] Z. Lu, E. Manias, and D. D. Macdonald, "Dielectric Relaxation Spectroscopy Studies on Water-Saturated Nafion 117 Membrane," in In Proceedings of the Electrochemical Society 204th National Meeting, 2003. 
[38] G. W. Gross and J. Johnson, “The Layered Capacitor Method for Dielectric Bridge Measurements. Data Analysis and Interpretation of Fluoride Doped ICE.," IEEE Trans. Electr. Insul., no. 5, pp. 485-497, 1983.

[39] A. R. Von Hippel, "Dielectrics and waves," 1954.

[40] J. M. Charlesworth, "Deconvolution of overlapping relaxations in dynamic mechanical spectra,” J. Mater. Sci., vol. 28, no. 2, pp. 399-404, 1993.

[41] S. Havriliak and S. Negami, "A complex plane analysis of $\alpha$-dispersions in some polymer systems," in Journal of Polymer Science Part C: Polymer Symposia, 1966, vol. 14, no. 1, pp. 99-117.

[42] S. Havriliak and S. Negami, "A complex plane representation of dielectric and mechanical relaxation processes in some polymers," Polymer (Guildf)., vol. 8, pp. 161-210, 1967.

[43] H. Eyring, "The activated complex in chemical reactions," J. Chem. Phys., vol. 3, no. 2, pp. 107-115, 1935.

[44] J. Heijboer, "Molecular origin of relaxations in polymers," Ann. N. Y. Acad. Sci., vol. 279, no. 1, pp. 104-116, 1976.

[45] J. Heijboer, "Secondary loss peaks in glassy amorphous polymers," in Molecular basis of transitions and relaxations, D. J. Meier, Ed. CRC Press, 1978, p. 429.

[46] H. Vogel, "The temperature dependence law of the viscosity of fluids," Phys. Z, vol. 22, pp. 645-646, 1921.

[47] G. S. Fulcher, "Analysis of recent measurements of the viscosity of glasses," $J$. Am. Ceram. Soc., vol. 75, no. 5, pp. 1043-1055, 1992.

[48] K. Kunal, C. G. Robertson, S. Pawlus, S. F. Hahn, and A. P. Sokolov, "Role of chemical structure in fragility of polymers: A qualitative picture," Macromolecules, vol. 41, no. 19, pp. 7232-7238, 2008.

[49] A. K. Doolittle, "Studies in Newtonian Flow. II. The Dependence of the Viscosity of Liquids on Free-Space,” J. Appl. Phys., vol. 22, no. 12, p. 1471, 1951. 
[50] A. K. Doolittle, "Studies in Newtonian Flow. III. The Dependence of the Viscosity of Liquids on Molecular Weight and Free Space (in Homologous Series)," J. Appl. Phys., vol. 23, no. 2, p. 236, 1952.

[51] H. Fujimori and M. Oguni, "Correlation index $(\operatorname{Tg} \alpha-\operatorname{Tg} \beta) \operatorname{Tg} \alpha$ and activation energy ratio $\Delta \epsilon \mathrm{a} \alpha \Delta \epsilon \mathrm{a} \beta$ as parameters characterizing the structure of liquid and glass," Solid State Commun., vol. 94, no. 2, pp. 157-162, 1995.

[52] M. . Cerrada, J. . Pereña, R. Benavente, and E. Pérez, "Viscoelastic processes in vinyl alcohol-ethylene copolymers. Influence of composition and thermal treatment," Polymer (Guildf)., vol. 41, no. 17, pp. 6655-6661, 2000.

[53] F. P. La Mantia and G. Spadaro, "Dielectric and Dynamic-mechanical behaviour of ethylene-vynil alcohol copolymers," Mater. Chem. Phys., pp. 315-321, 1983.

[54] I. Tantis, G. C. Psarras, and D. Tasis, "Functionalized graphene - poly(vinyl alcohol) nanocomposites: Physical and dielectric properties," Express Polym. Lett., vol. 6, no. 4, pp. 283-292, 2012.

[55] R. Popli, M. Glotin, L. Mandelkern, and R. S. Benson, "Dynamic mechanical studies of $\alpha$ and $\beta$ relaxations of polyethylenes," J. Polym. Sci. Polym. Phys. Ed., vol. 22, no. 3, pp. 407-448, Mar. 1984.

[56] T. Fujiki, M. Saito, M. Uemura, and Y. Kosaka, "On the secondary dispersion due to motion of chain branches in ethylene polymers," J. Polym. Sci. Part A-2 Polym. Phys., vol. 8, no. 1, pp. 153-159, Jan. 1970.

[57] S. De Petris, P. Laurienzo, M. Malinconico, M. Pracella, and M. Zendron, "Study of blends of nylon 6 with EVOH and carboxyl-modified EVOH and a preliminary approach to films for packaging applications," J. Appl. Polym. Sci., vol. 68 , no. 4, pp. 637-648, 1998.

[58] Z. Dang, L. Wang, Y. I. Yin, Q. Zhang, and Q. Lei, “Giant dielectric permittivities in functionalized carbon-nanotube/electroactive-polymer nanocomposites," Adv. Mater., vol. 19, no. 6, pp. 852-857, 2007.

[59] T. Tanaka, "Dielectric nanocomposites with insulating properties," IEEE Transactions on Dielectrics and Electrical Insulation, vol. 12, no. 5. pp. 914- 26 - 
928, 2005.

[60] R. Popli and L. Mandelkern, "The transition in ethylene copolymers: the $\beta$ transition," Polym. Bull., vol. 9, no. 6-7, pp. 260-267, 1983.

[61] K.-H. Illers, "Dynamic-mechanical behaviour and chemical transformations of polymer systems,” Eur. Polym. J., vol. 5, pp. 133IN3-147, 1969.

[62] W. . Macknight and R. . Tetreault, "Dynamic mechanical behavior of partially hydrolyzed ethylene-vinyl acetate copolymers," J Polym Sci Part C, vol. 35, pp. 117-127, 1971.

[63] E. Zuza, J. M. Ugartemendia, A. Lopez, E. Meaurio, A. Lejardi, and J. R. Sarasua, "Glass transition behavior and dynamic fragility in polylactides containing mobile and rigid amorphous fractions," Polymer (Guildf)., vol. 49, no. 20, pp. 4427-4432, 2008. 


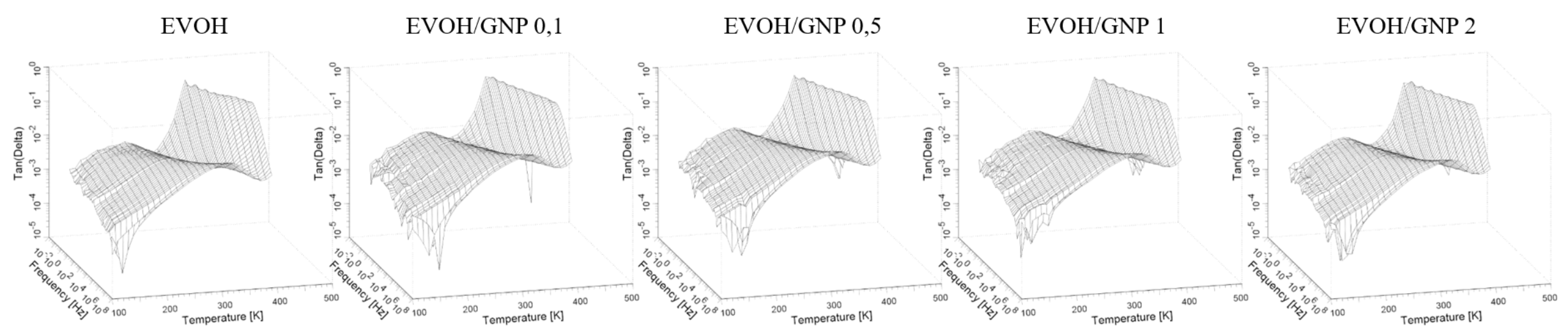

Figure 1. Tridimensional (3D) plot of the imaginary component of the loss tangent ( $\tan \delta$ ) for the electrospun poly(ethylene-co-vinyl alcohol) (EVOH) and EVOH/ graphene nanoplatelets (GNPs) nanofibers 

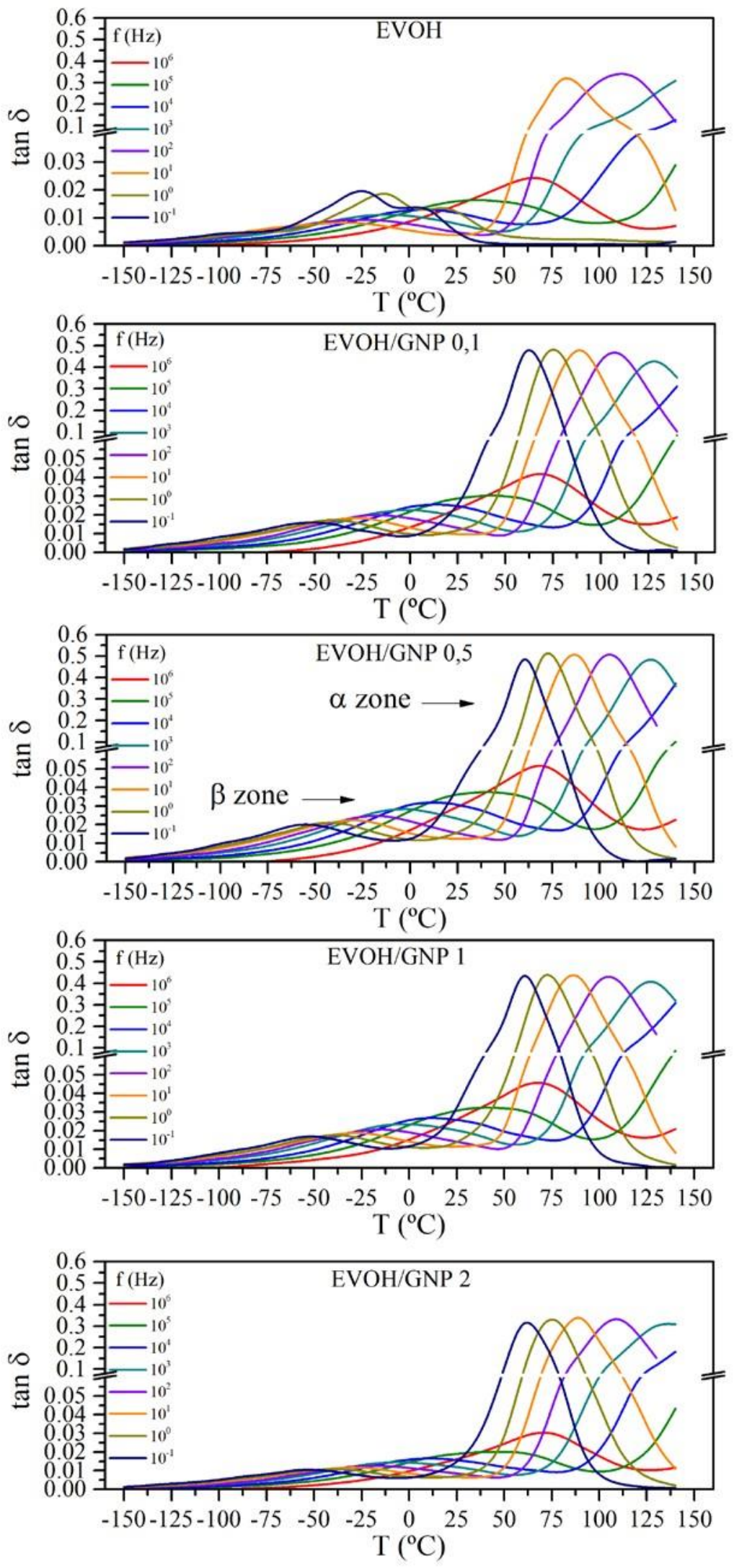

Figure 2. Isochronal curves of loss tangent $(\tan \delta)$ of the electrospun poly(ethylene-co-vinyl alcohol) $(\mathrm{EVOH})$ and $\mathrm{EVOH} /$ graphene nanoplatelets (GNPs) nanomats 


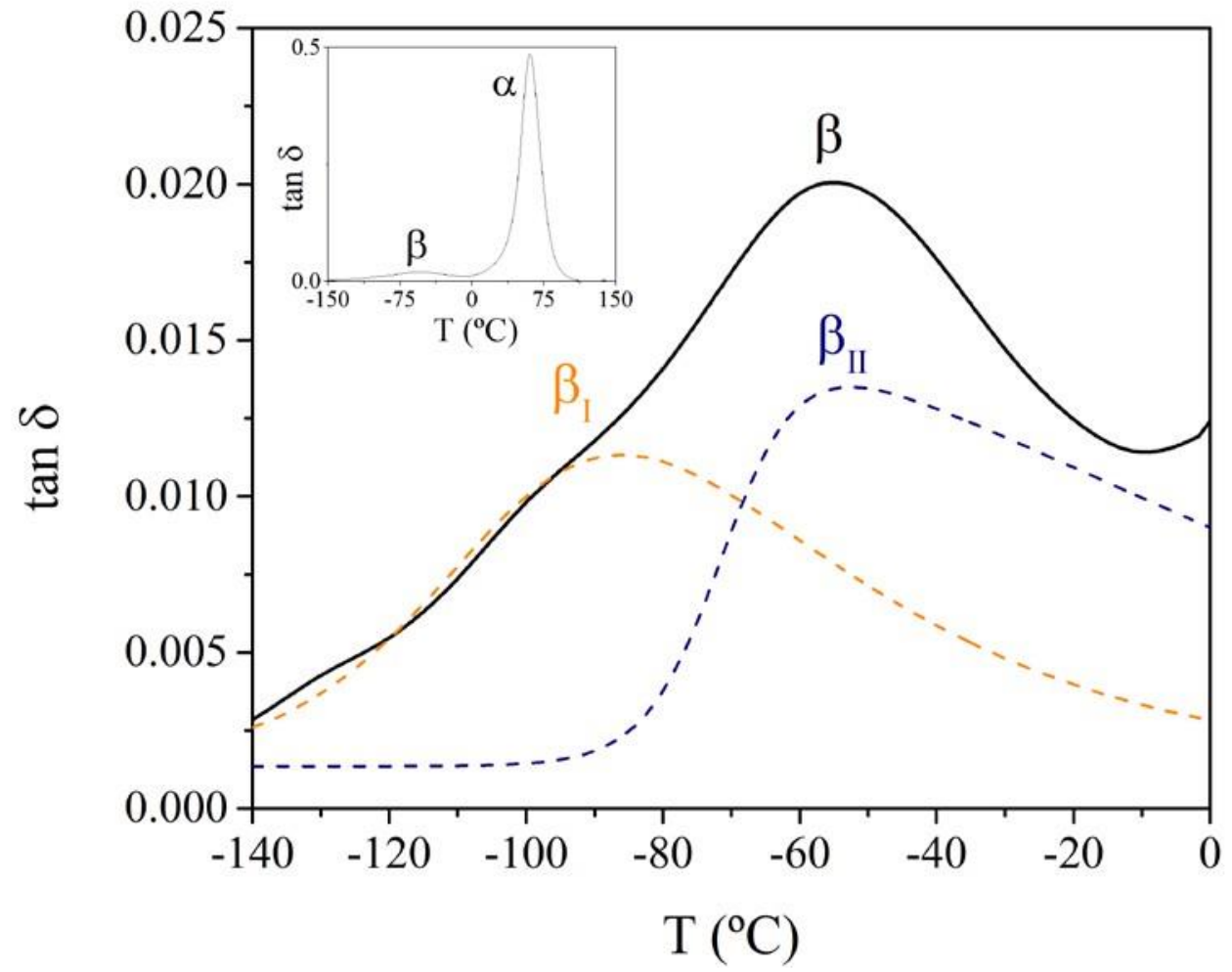

Figure 3. Deconvolution of $\beta$-zone by means of 2 Havriliak-Negami functions. Example for $\mathrm{EVOH} / \mathrm{GNP} 0.5 \mathrm{wt} \%$ at a frequency of $10^{-1} \mathrm{~Hz}$. Insert: Entire dielectric curve for this measurement. 


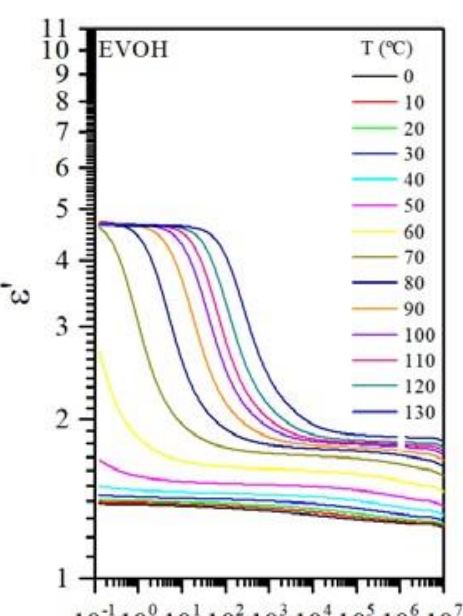

$10^{-1} 10^{0} 10^{1} 10^{2} 10^{3} 10^{4} 10^{5} 10^{6} 10^{7}$

$\mathrm{f}(\mathrm{Hz})$

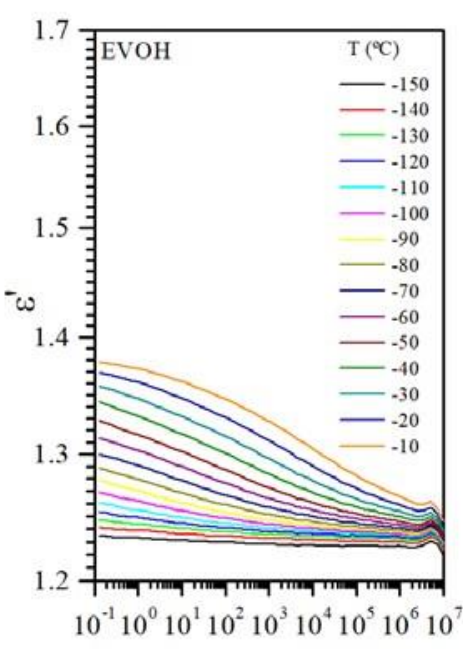

$\mathrm{f}(\mathrm{Hz})$

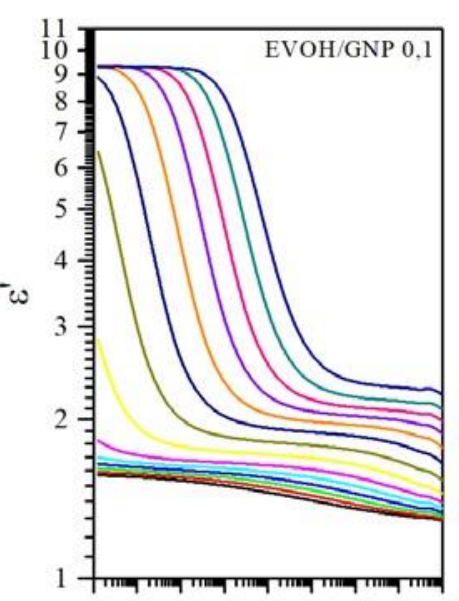

$10^{-1} 10^{0} 10^{1} 10^{2} 10^{3} 10^{4} 10^{5} 10^{6} 10$

$\mathrm{f}(\mathrm{Hz})$

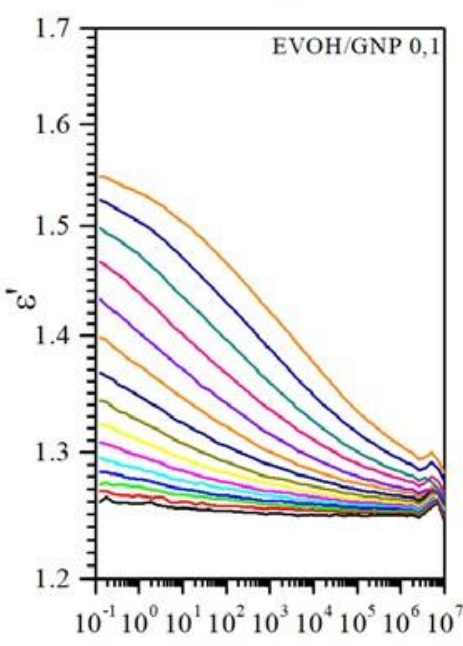

$\mathrm{f}(\mathrm{Hz})$

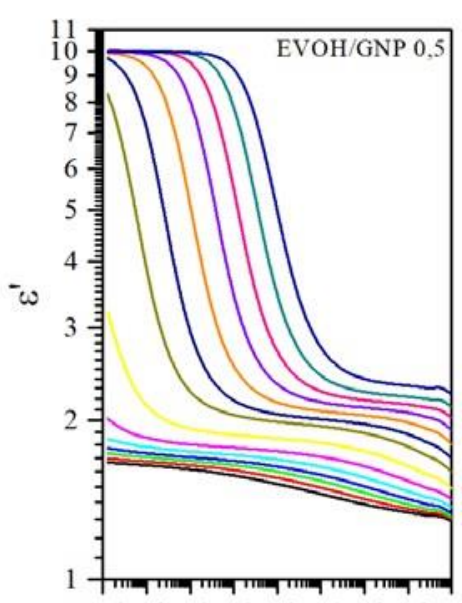

$10^{-1} 10^{0} 10^{1} 10^{2} 10^{3} 10^{4} 10^{5} 10^{6} 10$

$\mathrm{f}(\mathrm{Hz})$

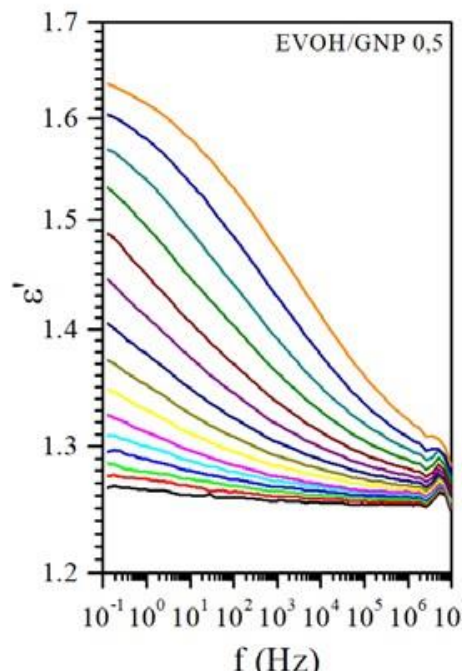

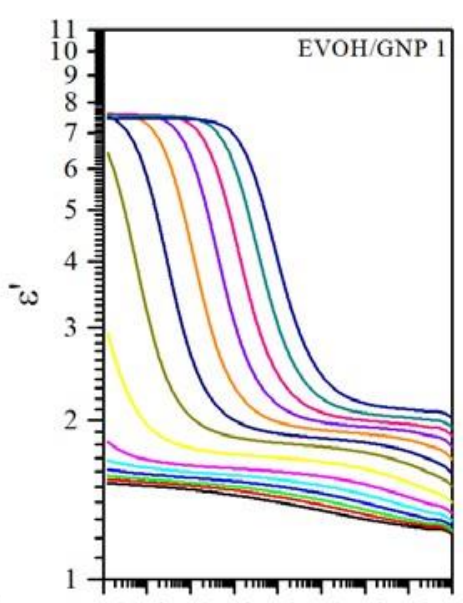

$10^{-1} 10^{0} 10^{1} 10^{2} 10^{3} 10^{4} 10^{5} 10^{6} 10^{7}$

$\mathrm{f}(\mathrm{Hz})$

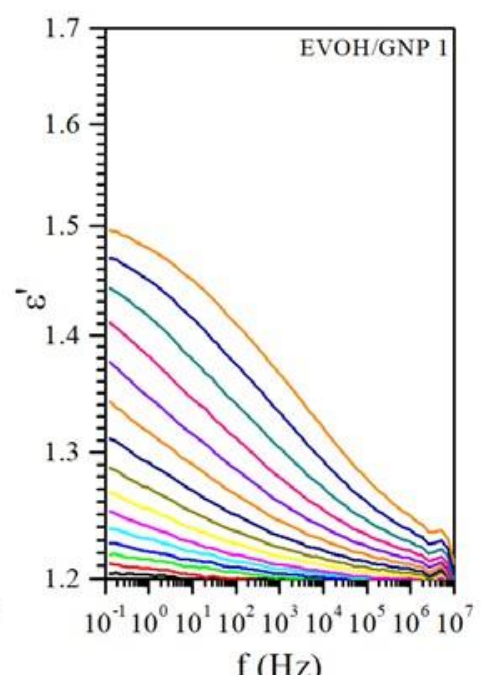

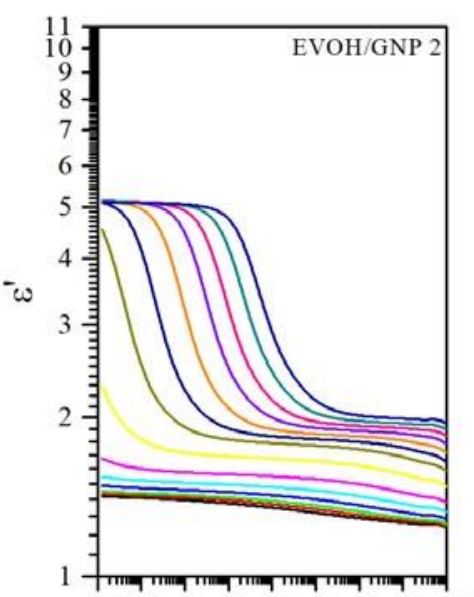

$10^{-1} 10^{0} 10^{1} 10^{2} 10^{3} 10^{4} 10^{5} 10^{6} 10^{7}$

$\mathrm{f}(\mathrm{Hz})$

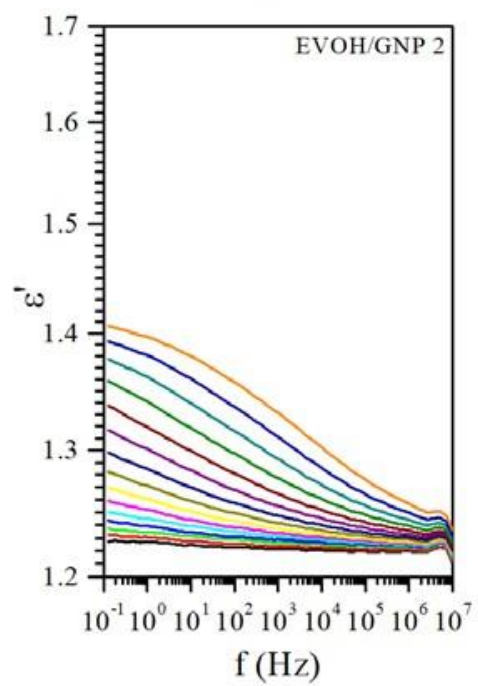

Figure 4. Frequency (f) response of the dielectric permittivity $\varepsilon$ ' of the electrospun poly(ethylene-co-vinyl alcohol) (EVOH) and EVOH/ graphene nanoplatelets (GNPs) nanofibers at temperature ranges between -150 and $-10{ }^{\circ} \mathrm{C}$ (down) and between 0 and $130{ }^{\circ} \mathrm{C}$ (up) 

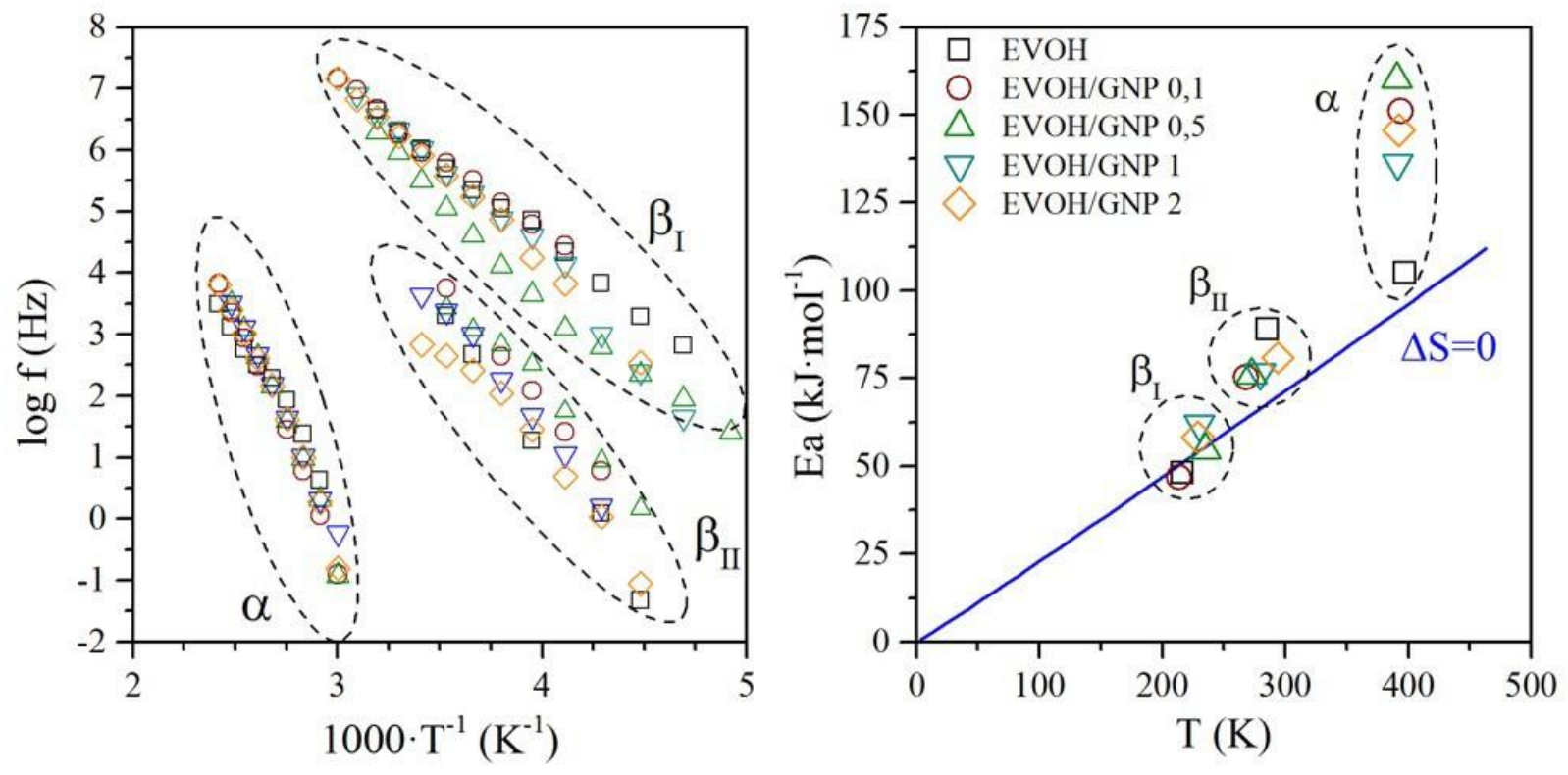

Figure 5. Arrhenius maps (left) and Eyring plot (right) of the dielectric relaxations of the electrospun poly(ethylene-co-vinyl alcohol) (EVOH) and $\mathrm{EVOH} /$ graphene nanoplatelets (GNPs) 


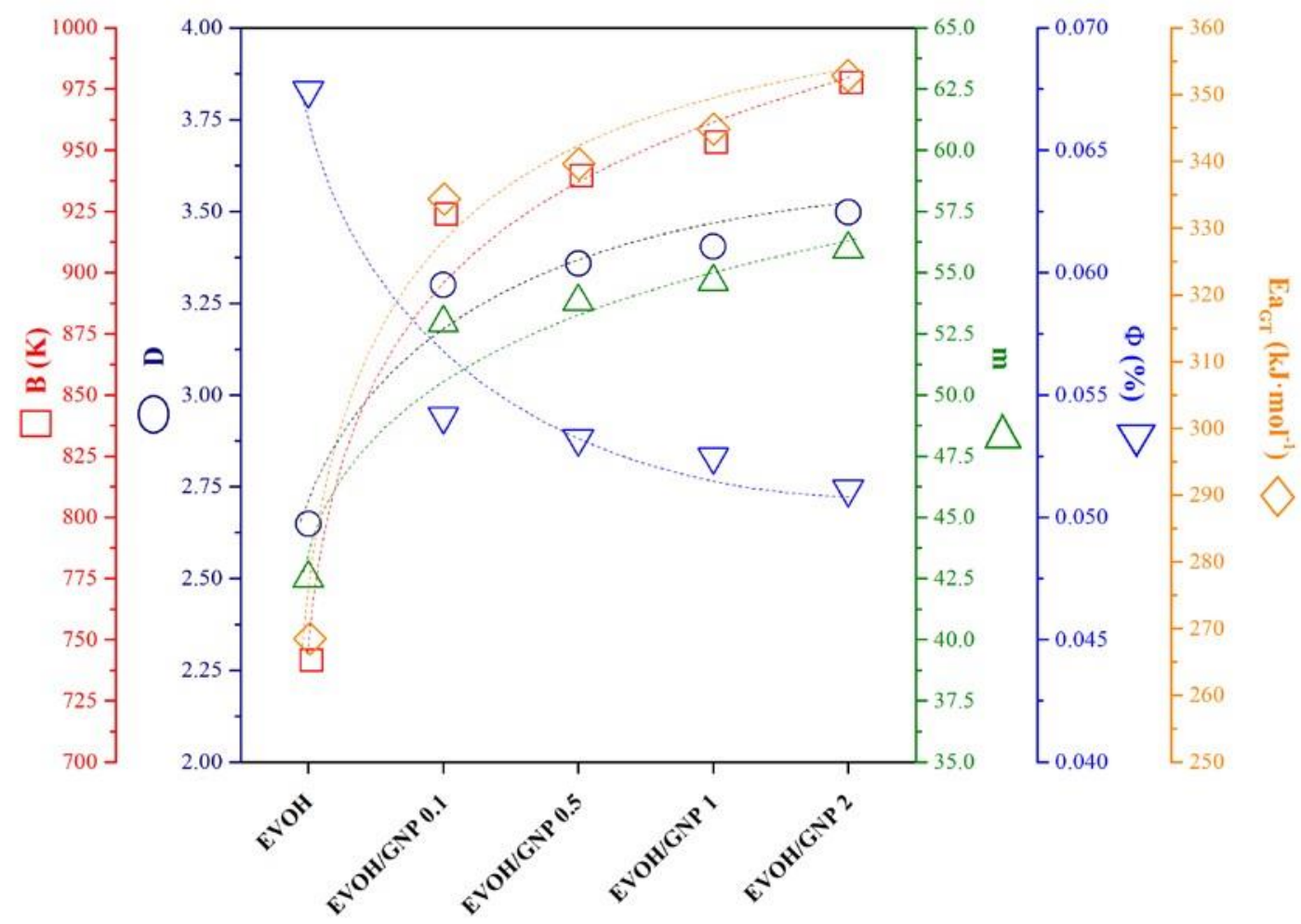

Figure 6. Evolution of the dynamic fragility indicators with the increment of graphene nanoplatelets (GNPs) in the electrospun poly(ethylene-co-vinyl alcohol) (EVOH) nanomats. Dotted lines are shown as an aid for the eye. 